EDITORIAL

\title{
La actividad física y su importancia en los niños
}

A nivel mundial, la obesidad casi se ha duplicado desde 1980. En 2014, el $10 \%$ de los hombres y el $14 \%$ de las mujeres de 18 años o más eran obesos. En el 2019 el número de niños menores de 5 años con sobrepeso fue de 39 millones, cerca de 340 millones de niños y adolescentes estaban en sobrepeso o tenían obesidad.'

Es por esto qué debemos como pediatras estimular la actividad física en nuestros pacientes, iniciando desde lactantes.

\section{¿A qué se denomina actividad física?}

A cualquier movimiento corporal producido por los músculos esqueléticos que exija gasto de energía. (Definición de OMS) o

Toda actividad o ejercicio que tenga como consecuencia el gasto de energía y que ponga en movimiento un montón de fenómenos a nivel corporal, psíquico y emocional en la persona que la realiza. La actividad física puede ser realizada de manera planeada y organizada o de manera espontánea o involuntaria, aunque en ambos casos los resultados son similares. ${ }^{2}$

\section{Tipos de actividades físicas que se recomiendan según la edad ${ }^{3}$}

Lactantes: Juegos en el suelo o en el agua. Movimientos de brazos y piernas, buscar y coger objetos, girar la cabeza, tirar, empujar y jugar con otras personas, patear, gatear, ponerse de pie, arrastrarse y andar.

Preescolares (hasta 5 años): Caminar, manejar bicicleta, columpiarse, correr, jugar a la pelota, saltar y natación.

Escolares y adolescentes: Actividad física moderada-intensa al menos 60 minutos al día, que se puede repartir en dos sesiones diarias, que se pueden intercalar con otros tipos de ejercicios que fortalezcan el sistema óseo y muscular de dos a tres veces a la semana. Lo principal es disminuir el sedentarismo.

\section{¿Qué beneficios tiene la actividad física?}

- Fortalece el organismo y evita enfermedades.

- Mejora la autoestima.

- Puede ayudar al bienestar mental (disminuir la depresión, ansiedad y estrés).

- Mejora la función cognitiva, la concentración y el rendimiento académico.

Permite que tanto el niño como el adolescente desarrollen competencias y cualidades, tales como el trabajo en equipo, disciplina, cooperación, comunicación y otras. ${ }^{4}$

\section{¿Cómo iniciar a los niños en actividades físicas?}

El desarrollo de la actividad y ejercicio físico debe ser visto por el niño como un momento para divertirse y jugar, por lo tanto es importante estimular las actividades en grupo (fútbol, natación, bicicleta, etc), al aire libre que permitirán un refuerzo positivo, consiguiendo que se mantengan como "hábito divertido". Se deben tomar las medidas de seguridad, como uso de casco, protector de brazos y rodillas, el lugar de juego debe ser adecuado y estar exento de peligros. 
¿Qué hacer en climas tropicales como el nuestro?

- Hidratación antes, durante y después de la actividad física.

- Entrenar por la mañana temprano o por la noche, cuando las temperaturas suelen ser menores.

- Reducir la duración, distancia y/o intensidad del entrenamiento cuando el calor y la humedad sean altos.

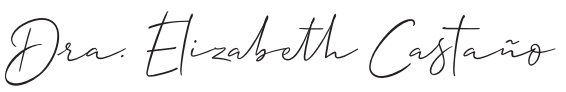

\section{Dra. Elizabeth Castaño Editora Jefe}

\section{Referencias}

1. Organización Mundial de la Salud. Obesidad y sobrepeso. Abril 2021

2. Martin A, Booth JN, Laird Y, Sproule J, Reilly JJ, Saunders DH. Physical activity, diet and other behavioural interventions for improving cognition and school achievement in children and adolescents with obesity or overweight. Cochrane Database Syst Rev. 2018 Jan 29;1(1):CD009728. doi: 10.1002/14651858.CD009728.pub3. Update in: Cochrane Database Syst Rev. 2018 Mar 02;3:CD009728. PMID: 29376563; PMCID: PMC6491168.

3. Cortés- Martín EM, Sánchez Ruiz-Cabello FJ, Prelnfad. En Familia. Deporte y actividad física en los niños. Asociación Española de Pediatría. 2015. España

4. Rodríguez Torres AF, Rodríguez Alvear JC, Guerrero Gallardo HI, Arias Moreno ER, Paredes Alvear AE, Chávez Vaca VA. Beneficios de la actividad física para niños y adolescentes en el contexto escolar. Rev Cubana Med Gen Integr [Internet]. 2020 Jun [citado 2021 Jun 04]; 36(2): e1535. Disponible en: http://scielo.sld.cu/scielo.php?script=sci_arttext\&pid=S0864-21252020000200010\&ln$\mathrm{g}=$ es. Epub 01-Jul-2020. 